\title{
Multiple Positive Solutions to Multipoint Boundary Value Problem for a System of Second-Order Nonlinear Semipositone Differential Equations on Time Scales
}

\author{
Gang Wu, ${ }^{1,2}$ Longsuo Li, ${ }^{1}$ Xinrong Cong, ${ }^{1}$ and Xiufeng Miao ${ }^{1}$ \\ ${ }^{1}$ Department of Mathematics, Harbin Institute of Technology, Harbin, Heilongjiang 150001, China \\ ${ }^{2}$ Basic Science, Harbin University of Commerce, Harbin, Heilongjiang 150076, China \\ Correspondence should be addressed to Longsuo Li; lilongsuo6982@126.com
}

Received 13 November 2012; Revised 31 January 2013; Accepted 31 January 2013

Academic Editor: Naseer Shahzad

Copyright (C) 2013 Gang Wu et al. This is an open access article distributed under the Creative Commons Attribution License, which permits unrestricted use, distribution, and reproduction in any medium, provided the original work is properly cited.

We study a system of second-order dynamic equations on time scales $\left(p_{1} u_{1}^{\nabla}\right)^{\Delta}(t)-q_{1}(t) u_{1}(t)+\lambda f_{1}\left(t, u_{1}(t), u_{2}(t)\right)=0, t \in$ $\left(t_{1}, t_{n}\right),\left(p_{2} u_{2}^{\nabla}\right)^{\Delta}(t)-q_{2}(t) u_{2}(t)+\lambda f_{2}\left(t, u_{1}(t), u_{2}(t)\right)=0$, satisfying four kinds of different multipoint boundary value conditions, $f_{i}$ is continuous and semipositone. We derive an interval of $\lambda$ such that any $\lambda$ lying in this interval, the semipositone coupled boundary value problem has multiple positive solutions. The arguments are based upon fixed-point theorems in a cone.

\section{Introduction}

In this paper, we consider the following dynamic equations on time scales:

$$
\begin{array}{r}
\left(p_{1} u_{1}^{\nabla}\right)^{\Delta}(t)-q_{1}(t) u_{1}(t)+\lambda f_{1}\left(t, u_{1}(t), u_{2}(t)\right)=0, \\
t \in\left(t_{1}, t_{n}\right), \lambda>0, \\
\left(p_{2} u_{2}^{\nabla}\right)^{\Delta}(t)-q_{2}(t) u_{2}(t)+\lambda f_{2}\left(t, u_{1}(t), u_{2}(t)\right)=0,
\end{array}
$$

satisfying one of the boundary value conditions

$$
\begin{gathered}
\alpha_{1} u_{1}\left(t_{1}\right)-\beta_{1} p_{1}\left(t_{1}\right) u_{1}^{\nabla}\left(t_{1}\right)=0, \\
\gamma_{1} u_{1}\left(t_{n}\right)+\delta_{1} p_{1}\left(t_{n}\right) u_{1}^{\nabla}\left(t_{n}\right)=\sum_{i=2}^{n-2} b_{i} u_{1}\left(t_{i}\right), \\
\alpha_{2} u_{2}\left(t_{1}\right)-\beta_{2} p_{2}\left(t_{1}\right) u_{2}^{\nabla}\left(t_{1}\right)=0, \\
\gamma_{2} u_{2}\left(t_{n}\right)+\delta_{2} p_{1}\left(t_{n}\right) u_{2}^{\nabla}\left(t_{n}\right)=\sum_{i=2}^{n-2} b_{i} u_{2}\left(t_{i}\right),
\end{gathered}
$$

$$
\begin{gathered}
\alpha_{1} u_{1}\left(t_{1}\right)-\beta_{1} p_{1}\left(t_{1}\right) u_{1}^{\nabla}\left(t_{1}\right)=\sum_{i=2}^{n-2} a_{i} u_{1}\left(t_{i}\right), \\
\gamma_{1} u_{1}\left(t_{n}\right)+\delta_{1} p_{1}\left(t_{n}\right) u_{1}^{\nabla}\left(t_{n}\right)=0, \\
\alpha_{2} u_{2}\left(t_{1}\right)-\beta_{2} p_{2}\left(t_{1}\right) u_{2}^{\nabla}\left(t_{1}\right)=\sum_{i=2}^{n-2} a_{i} u_{2}\left(t_{i}\right),
\end{gathered}
$$$$
\begin{aligned}
& \gamma_{2} u_{2}\left(t_{n}\right)+\delta_{2} p_{1}\left(t_{n}\right) u_{2}^{\nabla}\left(t_{n}\right)=0, \\
& \alpha_{1} u_{1}\left(t_{1}\right)-\beta_{1} p_{1}\left(t_{1}\right) u_{1}^{\nabla}\left(t_{1}\right)=0,
\end{aligned}
$$$$
\gamma_{1} u_{1}\left(t_{n}\right)+\delta_{1} p_{1}\left(t_{n}\right) u_{1}^{\nabla}\left(t_{n}\right)=\sum_{i=2}^{n-2} b_{i} u_{1}\left(t_{i}\right)
$$$$
\alpha_{2} u_{2}\left(t_{1}\right)-\beta_{2} p_{2}\left(t_{1}\right) u_{2}^{\nabla}\left(t_{1}\right)=\sum_{i=2}^{n-2} a_{i} u_{2}\left(t_{i}\right) \text {, }
$$$$
\gamma_{2} u_{2}\left(t_{n}\right)+\delta_{2} p_{1}\left(t_{n}\right) u_{2}^{\nabla}\left(t_{n}\right)=0,
$$$$
\alpha_{1} u_{1}\left(t_{1}\right)-\beta_{1} p_{1}\left(t_{1}\right) u_{1}^{\nabla}\left(t_{1}\right)=\sum_{i=2}^{n-2} a_{i} u_{1}\left(t_{i}\right) \text {, }
$$ 


$$
\begin{gathered}
\gamma_{1} u_{1}\left(t_{n}\right)+\delta_{1} p_{1}\left(t_{n}\right) u_{1}^{\nabla}\left(t_{n}\right)=0, \\
\alpha_{2} u_{2}\left(t_{1}\right)-\beta_{2} p_{2}\left(t_{1}\right) u_{2}^{\nabla}\left(t_{1}\right)=0, \\
\gamma_{2} u_{2}\left(t_{n}\right)+\delta_{2} p_{1}\left(t_{n}\right) u_{2}^{\nabla}\left(t_{n}\right)=\sum_{i=2}^{n-2} b_{i} u_{2}\left(t_{i}\right),
\end{gathered}
$$

where

$$
\begin{aligned}
& p_{i}, q_{i}:\left[t_{1}, t_{n}\right] \longrightarrow(0,+\infty) \\
& \quad \text { with } p_{i} \in C^{\Delta}\left[t_{1}, t_{n}\right), \quad q_{i} \in C\left[t_{1}, t_{n}\right] \quad \text { for } i=1,2 ; \\
& \alpha_{i}, \beta_{i}, \gamma_{i}, \delta_{i} \in[0,+\infty) \\
& \quad \text { with } \alpha_{i} \gamma_{i}+\alpha_{i} \delta_{i}+\beta_{i} \gamma_{i}>0 \text { for } i=1,2,
\end{aligned}
$$

and $f_{i}$ is continuously and nonegative functionsquad, $a_{i}, b_{i} \in$ $[0,+\infty)$ for $i \in\{1,2, \ldots, n\}$; the points $t_{i} \in \mathbb{T}_{\kappa}^{\kappa}$ for $i \in$ $\{1,2, \ldots, n\}$ with $t_{1}<t_{2}<\cdots<t_{n}$.

In the past few years, the boundary value problems of dynamic equations on time scales have been studied by many authors (see [1-19] and references). Recently, multipoint boundary value problems on time scale have been studied, for instance, see [1-12].

In 2006, Anderson and Ma [1] studied the second-order multiple time-scale eigenvalue problem:

$$
\begin{array}{r}
\left(p y^{\nabla}\right)^{\Delta}(t)-q(t) y(t)+\lambda h(t) f(y)=0, \\
t \in\left(t_{1}, t_{n}\right), \lambda>0, \\
\alpha y\left(t_{1}\right)-\beta p\left(t_{1}\right) y^{\nabla}\left(t_{1}\right)=\sum_{i=2}^{n-2} a_{i} y\left(t_{i}\right), \\
\gamma y\left(t_{n}\right)+\delta p\left(t_{n}\right) y^{\nabla}\left(t_{n}\right)=\sum_{i=2}^{n-2} b_{i} y\left(t_{i}\right),
\end{array}
$$

where the functions $f:[0,+\infty) \rightarrow[0,+\infty)$ and $h:$ $\left[t_{1}, t_{n}\right] \rightarrow[0,+\infty)$ are continuous. The authors discuss conditions for the existence of at least one positive solution to the second-order Sturm-Liouville-type multiple eigenvalue problem on time scales.

In 2009, Feng et al. [2] studied

$$
\begin{aligned}
\left(p y^{\nabla}\right)^{\Delta}(t)-q(t) y(t) & =f(t, y), \\
t & \in\left(t_{1}, t_{n}\right), \\
\alpha y\left(t_{1}\right)-\beta p\left(t_{1}\right) y^{\nabla}\left(t_{1}\right) & =\sum_{i=2}^{n-2} a_{i} y\left(t_{i}\right), \\
\gamma y\left(t_{n}\right)+\delta p\left(t_{n}\right) y^{\nabla}\left(t_{n}\right) & =\sum_{i=2}^{n-2} b_{i} y\left(t_{i}\right),
\end{aligned}
$$

where the functions $f(t, y)=\sum_{j=1}^{n} c_{j}(t) y^{v_{j}}, c_{j} \in C\left(\left[t_{1}, t_{n}\right]\right.$, $[0, \infty)), v_{i} \in[0, \infty), j=1,2, \ldots, n$. This paper shows the existence of multiple positive solutions for the boundary value problem on time scales.

In 2009, Topal and Yantir [3] studied the second-order nonlinear $m$-point boundary value problems

$$
\begin{gathered}
u^{\Delta \nabla}(t)+a(t) u^{\Delta}(t)+b(t) u(t) \\
+\lambda q(t) f(t, u(t))=0, \quad t \in(0,1)_{\mathbb{T}}, \\
u(\rho(0))=0, \quad u(\sigma(1))=\sum_{i=1}^{m-2} \alpha_{i} u\left(\eta_{i}\right),
\end{gathered}
$$

where $\alpha_{i} \geq 0,0<\eta_{i}<\eta_{i+1}<1$; for all $i=1,2, \ldots, m-$ $2 ; a \in C([0,1],[0,+\infty)), b \in C([0,1],(-\infty, 0]), f, q$ are continuously and nonegative functions. The authors deal with determining the value of $\lambda$, and the existences of multiple positive solutions of the equation are obtained. In 2010, Yuan and Liu [4] also study the second-order $m$-point boundary value problems; Yuan and Liu shows the existence of multiple positive solutions if $f$ is semipositone and superlinear.

Motivated by the above results mentioned, we study the second-order nonlinear $m$-point boundary value problem (1) with boundary condition $(k)$, and nonlinear term may be singularity and semipositone.

In this paper, the nonlinear term $f_{i}$ of (1) is suit to and semipositone and the superlinear case, we shall prove our two existence results for the problem (1) with $(k)$ by using a nonlinear alternative of Leray-Schauder type and Krasnosel'skii fixed-point theorem. This paper is organized as follows. In Section 2, we start with some preliminary lemmas. In Section 3, we give the main result which state the sufficient conditions for (1) with $m$-point boundary value $(k)$ to have existence of positive solutions $(k=2, \ldots, 5)$.

\section{Preliminaries}

In this section, we state the preliminary information that we need to prove the main results.

In this paper, for our constructions, we shall consider the Banach space $E=C\left[\rho\left(t_{1}\right), t_{n}\right]$ equipped with standard norm $\|x\|=\max _{\rho\left(t_{1}\right) \leq t \leq t_{n}}|x(t)|, x \in E$; for each $(x, y) \in E \times E$, we write $\|(x, y)\|_{1}=\|x\|+\|y\|$. Clearly, $\left(E \times E,\|\cdot\|_{1}\right)$ is a Banach space. Denote by $\phi_{i 1}$ and $\phi_{i 2}(i=1,2)$, the solutions of the equation

$$
\left(p_{i} u_{i}^{\nabla}\right)^{\Delta}(t)-q_{i}(t) u_{i}(t)=0, \quad t \in\left[t_{1}, t_{n}\right),
$$

under the initial conditions

$$
\begin{array}{ll}
u_{i}\left(t_{1}\right)=\beta_{i}, & p_{i}\left(t_{1}\right) u_{i}^{\nabla}\left(t_{1}\right)=\alpha_{i}, \\
u_{i}\left(t_{n}\right)=\delta_{i}, & p_{i}\left(t_{n}\right) u_{i}^{\nabla}\left(t_{n}\right)=-\gamma_{i},
\end{array}
$$


respectively. So that $\phi_{i 1}$ and $\phi_{i 2}(i=1,2)$ satisfy

$$
\begin{aligned}
\left(p_{i} \phi_{i 1}^{\nabla}\right)^{\Delta}(t)-q_{i}(t) \phi_{i 1}(t) & =0, \\
t \in\left[t_{1}, t_{n}\right), \quad \phi_{i 1}\left(t_{1}\right) & =\beta_{i}, \\
p_{i}\left(t_{1}\right) \phi_{i 1}^{\nabla}\left(t_{1}\right) & =\alpha_{i}, \\
\left(p_{i} \phi_{i 2}^{\nabla}\right)^{\Delta}(t)-q_{i}(t) \phi_{i 2}(t) & =0, \\
t \in\left[t_{1}, t_{n}\right), \quad \phi_{i 2}\left(t_{n}\right) & =\delta_{i}, \\
p_{i}\left(t_{n}\right) \phi_{i 2}^{\nabla}\left(t_{n}\right) & =-\gamma_{i},
\end{aligned}
$$

respectively. For $i=1,2$, set $d_{i}=\alpha_{i} \phi_{i 2}\left(t_{1}\right)-\beta_{i} p_{i}\left(t_{1}\right) \phi_{i 2}^{\nabla}\left(t_{1}\right)=$ $\gamma_{i} \phi_{i 1}\left(t_{n}\right)+\delta_{i} p_{i}\left(t_{n}\right) \phi_{i 1}^{\nabla}\left(t_{n}\right)$, the Green's function of the corresponding homogeneous boundary value problem is defined by

$$
\begin{aligned}
G_{i}(t, s) \\
\quad=\frac{1}{d_{i}}\left\{\begin{array}{ll}
\phi_{i 2}(t) \phi_{i 1}(s), & \rho\left(t_{1}\right) \leq s \leq t \leq t_{n}, \\
\phi_{i 1}(t) \phi_{i 2}(s), & \rho\left(t_{1}\right) \leq t \leq s \leq t_{n},
\end{array} \quad \text { for } i=1,2 .\right.
\end{aligned}
$$

From Lemmas 3.1 and 3.3 in [1], we have the following lemma.

Lemma 1. If $d_{i} \neq 0,\left(u_{1}, u_{2}\right)$ is a solution of (1) with boundary value condition $(k)$ if only and if

$$
\begin{array}{r}
u_{1}(t)=\lambda \int_{t_{1}}^{t_{n}} H_{1 k}(t, s) f_{1}\left(s, u_{1}(s), u_{2}(s)\right) \Delta s, \\
t \in\left[\rho\left(t_{1}\right), t_{n}\right], \\
u_{2}(t)=\lambda \int_{t_{1}}^{t_{n}} H_{2 k}(t, s) f_{2}\left(s, u_{1}(s), u_{2}(s)\right) \Delta s,
\end{array}
$$

where $k=2, \ldots, 5$, and

$$
\begin{array}{r}
H_{i 2}(t, s) \\
=G_{i}(t, s)+\frac{1}{d_{i}-\sum_{j=2}^{n-1} b_{j} \phi_{i 1}\left(t_{j}\right)} \sum_{j=2}^{n-1} b_{j} G_{i}\left(t_{j}, s\right) \phi_{i 1}(t), \\
\quad(i=1,2), \\
H_{i 3}(t, s) \\
=G_{i}(t, s)+\frac{1}{d_{i}-\sum_{j=2}^{n-1} a_{j} \phi_{i 2}\left(t_{j}\right)} \sum_{j=2}^{n-1} a_{j} G_{i}\left(t_{j}, s\right) \phi_{i 2}(t), \\
\quad(i=1,2),
\end{array}
$$

$$
\begin{aligned}
& H_{14}(t, s) \\
& =G_{1}(t, s)+\frac{1}{d_{1}-\sum_{j=2}^{n-1} b_{i} \phi_{11}\left(t_{i}\right)} \sum_{j=2}^{n-1} b_{i} G_{1}\left(t_{i}, s\right) \phi_{11}(t) \\
& =H_{12}(t, s), \\
& H_{24}(t, s) \\
& =G_{2}(t, s)+\frac{1}{d_{2}-\sum_{j=2}^{n-1} a_{i} \phi_{22}\left(t_{i}\right)} \sum_{j=2}^{n-1} a_{i} G_{2}\left(t_{i}, s\right) \phi_{22}(t) \\
& =H_{23}(t, s), \\
& H_{15}(t, s) \\
& =G_{1}(t, s)+\frac{1}{d_{1}-\sum_{j=2}^{n-1} a_{i} \phi_{12}\left(t_{i}\right)} \sum_{j=2}^{n-1} a_{i} G_{1}\left(t_{i}, s\right) \phi_{12}(t) \\
& =H_{13}(t, s), \\
& H_{25}(t, s) \\
& =G_{2}(t, s)+\frac{1}{d_{2}-\sum_{j=2}^{n-1} b_{i} \phi_{21}\left(t_{i}\right)} \sum_{j=2}^{n-1} b_{i} G_{2}\left(t_{i}, s\right) \phi_{21}(t) \\
& =H_{22}(t, s) \text {. }
\end{aligned}
$$

For the rest of the paper, we need the following assumption:

$$
0<\sum_{j=2}^{n-1} b_{j} \phi_{i 1}\left(t_{j}\right), \quad \sum_{j=2}^{n-1} a_{j} \phi_{i 2}\left(t_{j}\right)<d_{i}, \quad \text { for } i=1,2
$$

From $\phi_{i 1}$ is nondecreasing on $\left[\rho\left(t_{1}\right), t_{n}\right], \phi_{i 2}$ is nonincreasing on $\left[\rho\left(t_{1}\right), t_{n}\right]$ (see $[2$, Proposition 2.3]), it is easy to verify the following inequalities:

$$
\begin{gathered}
d_{i} G_{i}(t, s) \leq \phi_{i 1}(t) \phi_{i 2}(t), \\
d_{i} G_{i}(t, s) \leq \phi_{i 1}(s) \phi_{i 2}(s), \\
d_{i} G_{i}(t, s) \geq \frac{1}{\left\|\phi_{i 1}\right\|\left\|\phi_{i 2}\right\|} \phi_{i 1}(t) \phi_{i 2}(t) \phi_{i 1}(s) \phi_{i 2}(s) .
\end{gathered}
$$

Lemma 2. The Green's function $G_{i}(t, s)$ has properties

$$
G_{i}(t, s) \leq G_{i}(t, t)
$$

$$
\frac{d_{i}}{\left\|\phi_{i 1}\right\|\left\|\phi_{i 2}\right\|} G_{i}(t, t) G_{i}(s, s) \leq G_{i}(t, s) \leq G_{i}(s, s) .
$$


Lemma 3. For $H_{i k}(t, s), k=2, \ldots, 5$ and $i=1,2$, one has the conclusions $H_{i k}(t, s) \leq C^{*} G_{i}(s, s)$ and

$$
\begin{gathered}
c_{*} \phi_{i 1}(t) G_{i}(s, s) \leq H_{i 2}(t, s) \leq C^{*} \phi_{i 1}(t), \quad(i=1,2), \\
c_{*} \phi_{i 2}(t) G_{i}(s, s) \leq H_{i 3}(t, s) \leq C^{*} \phi_{i 2}(t), \quad(i=1,2), \\
c_{*} \phi_{i i}(t) G_{1}(s, s) \leq H_{i 4}(t, s) \leq C^{*} \phi_{i i}(t), \quad(i=1,2), \\
c_{*} \phi_{12}(t) G_{1}(s, s) \leq H_{15}(t, s) \leq C^{*} \phi_{12}(t), \\
c_{*} \phi_{21}(t) G_{1}(s, s) \leq H_{25}(t, s) \leq C^{*} \phi_{21}(t),
\end{gathered}
$$

where $C^{*}=C_{1}+C_{2}$ and

$$
\begin{aligned}
& C_{1}=\max _{i=1,2}\left\{1+\frac{\left\|\phi_{i 1}\right\|}{d_{i}-\sum_{j=2}^{n-1} b_{j} \phi_{i 1}\left(t_{j}\right)} \sum_{i=1}^{n-1} b_{j}\right. \\
& \left.+\frac{\left\|\phi_{i 2}\right\|}{d_{i}-\sum_{j=2}^{n-1} a_{j} \phi_{i 2}\left(t_{j}\right)} \sum_{j=2}^{n-1} a_{j}\right\}, \\
& C_{2}=\max _{i=1,2}\left\{\frac{\left\|\phi_{i 1}\right\|+\left\|\phi_{i 2}\right\|}{d_{i}}+\frac{1}{d_{i}-\sum_{j=2}^{n-1} b_{j} \phi_{i 1}\left(t_{j}\right)}\right. \\
& \times \sum_{j=2}^{n-1} b_{j} G_{i}\left(t_{j}, t_{j}\right)+\frac{1}{d_{i}-\sum_{j=2}^{n-1} b_{j} \phi_{i 2}\left(t_{j}\right)} \\
& \left.\times \sum_{j=2}^{n-1} a_{j} G_{2}\left(t_{j}, t_{j}\right)\right\}, \\
& c_{*}=\min \left\{\frac{d_{i}}{\left\|\phi_{i 1}\right\|\left\|\phi_{i 2}\right\|} \frac{1}{d_{i}-\sum_{j=2}^{n-1} b_{j} \phi_{i 1}\left(t_{j}\right)}\right. \\
& \times \sum_{j=2}^{n-1} b_{j} G_{i}\left(t_{j}, t_{j}\right), \\
& \frac{d_{i}}{\left\|\phi_{i 1}\right\|\left\|\phi_{i 2}\right\|} \frac{1}{d_{i}-\sum_{j=2}^{n-1} a_{j} \phi_{i 2}\left(t_{j}\right)} \\
& \left.\times \sum_{j=2}^{n-1} a_{j} G_{i}\left(t_{j}, t_{j}\right) ; i=1,2\right\} .
\end{aligned}
$$

Proof. From Lemma 2 and

$$
\begin{aligned}
& \frac{1}{d_{i}-\sum_{j=2}^{n-1} b_{j} \phi_{i 1}\left(t_{j}\right)} \sum_{j=2}^{n-1} b_{j} G_{i}\left(t_{j}, s\right) \phi_{i 1}(t) \\
& \leq \frac{1}{d_{i}-\sum_{j=2}^{n-1} b_{j} \phi_{i 1}\left(t_{j}\right)} \sum_{j=2}^{n-1} b_{j} G_{i}(s, s)\left\|\phi_{i 1}\right\|,
\end{aligned}
$$

$$
\begin{aligned}
& \frac{1}{d_{i}-\sum_{j=2}^{n-1} a_{j} \phi_{i 2}\left(t_{j}\right)} \sum_{j=2}^{n-1} a_{j} G_{i}\left(t_{j}, s\right) \phi_{i 2}(t) \\
& \leq \frac{1}{d_{i}-\sum_{j=2}^{n-1} a_{j} \phi_{i 2}\left(t_{j}\right)} \sum_{j=2}^{n-1} a_{j} G_{i}(s, s)\left\|\phi_{i 2}\right\|,
\end{aligned}
$$

we have

$$
H_{i k}(t, s) \leq C_{1} G_{i}(s, s) \leq C^{*} G_{i}(s, s) .
$$

For $k=2$ or 3 , we have

$$
\begin{aligned}
H_{i 2}(t, s) \leq & \frac{\left\|\phi_{i 2}(t)\right\|}{d_{i}} \phi_{i 1}(t)+\frac{1}{d_{i}-\sum_{j=2}^{n-1} b_{i} \phi_{i 1}\left(t_{i}\right)} \\
& \times \sum_{j=2}^{n-1} b_{j} G_{i}\left(t_{j}, t_{j}\right) \phi_{i 1}(t) \\
\leq & C^{*} \phi_{i 1}(t), \\
H_{i 3}(t, s) \leq & \frac{\left\|\phi_{i 1}(t)\right\|}{d_{i}} \phi_{i 2}(t)+\frac{1}{d_{i}-\sum_{j=2}^{n-1} a_{i} \phi_{i 2}\left(t_{i}\right)} \\
& \times \sum_{j=2}^{n-1} a_{j} G_{i}\left(t_{j}, t_{j}\right) \phi_{i 2}(t) \\
\leq & C^{*} \phi_{i 2}(t),
\end{aligned}
$$

$$
\begin{aligned}
H_{i 2}(t, s) \geq & \frac{1}{d_{i}-\sum_{j=2}^{n-1} b_{j} \phi_{i 1}\left(t_{j}\right)} \\
& \times \sum_{j=2}^{n-1} b_{j} G_{i}\left(t_{j}, s\right) \phi_{i 1}(t) \\
\geq & \frac{1}{d_{i}-\sum_{j=2}^{n-1} b_{j} \phi_{i 1}\left(t_{j}\right)} \\
& \times \sum_{j=2}^{n-1} b_{j} \frac{d_{i}}{\left\|\phi_{1}\right\|\left\|\phi_{2}\right\|} G_{i}\left(t_{j}, t_{j}\right) G_{i}(s, s) \phi_{i 1}(t) \\
\geq & c_{*} \phi_{i 1}(t) G_{i}(s, s),
\end{aligned}
$$

$$
\begin{aligned}
H_{i 3}(t, s) \geq & \frac{1}{d_{i}-\sum_{j=2}^{n-1} a_{j} \phi_{i 2}\left(t_{j}\right)} \\
& \times \sum_{j=2}^{n-1} a_{j} G_{i}\left(t_{j}, s\right) \phi_{i 2}(t) \\
\geq & \frac{1}{d_{i}-\sum_{j=2}^{n-1} a_{j} \phi_{i 1}\left(t_{j}\right)}
\end{aligned}
$$




$$
\begin{aligned}
& \times \sum_{j=2}^{n-1} a_{j} \frac{d_{i}}{\left\|\phi_{1}\right\|\left\|\phi_{2}\right\|} G_{i}\left(t_{j}, t_{j}\right) G_{i}(s, s) \phi_{i 2}(t) \\
\geq & c_{*} \phi_{i 2}(t) G_{i}(s, s) .
\end{aligned}
$$

So, we have

$$
\begin{gathered}
c_{*} \phi_{i 1}(t) G_{i}(s, s) \leq H_{i 2}(t, s) \leq C^{*} \phi_{i 1}(t), \quad(i=1,2), \\
c_{*} \phi_{i 2}(t) G_{i}(s, s) \leq H_{i 3}(t, s) \leq C^{*} \phi_{i 2}(t), \quad(i=1,2) . \\
\quad \text { Since } H_{14}(t, s)=H_{12}(t, s), H_{24}(t, s)=H_{23}(t, s), H_{15}(t, \\
s)=H_{13}(t, s), H_{25}(t, s)=H_{22}(t, s), \text { then we also have } \\
c_{*} \phi_{11}(t) G_{1}(s, s) \leq H_{14}(t, s)=H_{12}(t, s) \leq C^{*} \phi_{11}(t), \\
c_{*} \phi_{22}(t) G_{1}(s, s) \leq H_{24}(t, s)=H_{23}(t, s) \leq C^{*} \phi_{22}(t), \\
c_{*} \phi_{12}(t) G_{1}(s, s) \leq H_{15}(t, s)=H_{13}(t, s) \leq C^{*} \phi_{12}(t), \\
c_{*} \phi_{21}(t) G_{1}(s, s) \leq H_{25}(t, s)=H_{22}(t, s) \leq C^{*} \phi_{21}(t) .
\end{gathered}
$$

The proof is complete.

The following theorems will play a major role in our next analysis.

Theorem 4 (see [20]). Let $X$ be a Banach space, and $\Omega \subset X$ closed and convex. Assume $U$ is a relatively open subset of $\Omega$ with $0 \in U$, and let $S: \bar{U} \rightarrow \Omega$ be a compact, continuous map. Then either

(1) S has a fixed point in $\bar{U}$, or

(2) there exists $u \in \partial U$ and $v \in(0,1)$, with $u=\nu S u$.

Theorem 5 (see [21]). Let $X$ be a Banach space, and let $P \subset X$ be a cone in $X$. Let $\Omega_{1}, \Omega_{2}$ be bounded open subsets of $X$ with $0 \in \Omega_{1} \subset \bar{\Omega}_{1} \subset \Omega_{2}$, and let $S: P \rightarrow P$ be a completely continuous operator such that, either

(1) $\|S w\| \leq\|w\|, w \in P \cap \partial \Omega_{1},\|S w\| \geq\|w\|, w \in P \cap \partial \Omega_{2}$, or

(2) $\|S w\| \geq\|w\|, w \in P \cap \partial \Omega_{1},\|S w\| \leq\|w\|, w \in P \cap \partial \Omega_{2}$.

Then $S$ has a fixed point in $P \cap \bar{\Omega}_{2} \backslash \Omega_{1}$.

\section{Main Results}

We make the following assumptions:

$\left(\mathrm{H}_{1}\right) f_{i}\left(t, u_{1}, u_{2}\right) \in C\left(\left[t_{1}, t_{n}\right] \times[0,+\infty)^{2},(-\infty,+\infty)\right)$, moreover there exists a function $g(t) \in L^{1}\left(\left[t_{1}, t_{n}\right],(0\right.$, $+\infty)$ ) such that $f_{i}\left(t, u_{1}, u_{2}\right) \geq-g(t)$, for any $t \in$ $\left(t_{1}, t_{n}\right), u_{i} \in[0,+\infty), i=1,2$.

$\left(\mathrm{H}_{1}^{*}\right) f_{i}\left(t, u_{1}, u_{2}\right) \in C\left(\left(t_{1}, t_{n}\right) \times[0,+\infty)^{2},(-\infty,+\infty)\right)$ may be singular at $t=t_{1}, t_{n}$; moreover, there exists a function $g(t) \in L^{1}\left(\left(t_{1}, t_{n}\right),(0,+\infty)\right)$ such that $f_{i}\left(t, u_{1}\right.$, $\left.u_{2}\right) \geq-g(t)$, for any $t \in\left(t_{1}, t_{n}\right), u_{i} \in[0,+\infty)$.

$\left(\mathrm{H}_{2}\right) f_{i}(t, 0,0)>0$, for $t \in\left[t_{1}, t_{n}\right](i=1,2)$.
$\left(\mathrm{H}_{3}\right)$ There exists $\left[\theta_{1}, \theta_{2}\right] \quad \subset \quad\left(t_{1}, t_{n}\right)$ such that $\lim _{u_{1}+u_{2} \uparrow+\infty} \min _{t \in\left[\theta_{1}, \theta_{2}\right]}\left(f_{i}\left(t, u_{1}, u_{2}\right) /\left(u_{1}+u_{2}\right)\right)=$ $+\infty(i=1,2)$.

$\left(\mathrm{H}_{4}\right) \int_{t_{1}}^{t_{n}} G_{i}(s, s) g(s) \nabla s<+\infty$ and $\int_{t_{1}}^{t_{n}} G_{i}(s, s) f_{i}\left(s, z_{1}\right.$, $\left.z_{2}\right) \nabla s<+\infty$ for any $z_{i} \in[0, m], m>0$ is any constant $(i=1,2)$.

In fact, we only consider the system

$$
\begin{gathered}
\left(p_{1} x_{1}^{\nabla}\right)^{\Delta}(t)-q_{1}(t) x_{1}(t)+\lambda\left(f _ { 1 } \left(t,\left[x_{1}(t)-v_{1 k}(t)\right]^{*},\right.\right. \\
\left.\left[x_{2}(t)-v_{2 k}(t)\right]^{*}\right) \\
+g(t))=0, \\
\lambda>0, \\
\left(p_{2} x_{2}^{\nabla}\right)^{\Delta}(t)-q_{2}(t) x_{2}(t)+\lambda\left(f _ { 2 } \left(t,\left[x_{1}(t)-v_{1 k}(t)\right]^{*},\right.\right. \\
\left.\left[x_{2}(t)-v_{2 k}(t)\right]^{*}\right) \\
+g(t))=0 \\
\lambda>0
\end{gathered}
$$

with one of the boundary value conditions

$$
\begin{gathered}
\alpha_{1} x_{1}\left(t_{1}\right)-\beta_{1} p_{1}\left(t_{1}\right) x_{1}^{\nabla}\left(t_{1}\right)=0, \\
\gamma_{1} x_{1}\left(t_{n}\right)+\delta_{1} p_{1}\left(t_{n}\right) x_{1}^{\nabla}\left(t_{n}\right)=\sum_{i=2}^{n-2} b_{i} x_{1}\left(\eta_{i}\right), \\
\alpha_{2} x_{2}\left(t_{1}\right)-\beta_{2} p_{2}\left(t_{1}\right) x_{2}^{\nabla}\left(t_{1}\right)=0, \\
\gamma_{2} x_{2}\left(t_{n}\right)+\delta_{2} p_{1}\left(t_{n}\right) x_{2}^{\nabla}\left(t_{n}\right)=\sum_{i=2}^{n-2} b_{i} x_{2}\left(\eta_{i}\right), \\
\alpha_{1} x_{1}\left(t_{1}\right)-\beta_{1} p_{1}\left(t_{1}\right) x_{1}^{\nabla}\left(t_{1}\right)=\sum_{i=2}^{n-2} a_{i} x_{1}\left(\eta_{i}\right), \\
\gamma_{1} x_{1}\left(t_{n}\right)+\delta_{1} p_{1}\left(t_{n}\right) x_{1}^{\nabla}\left(t_{n}\right)=0, \\
\alpha_{2} x_{2}\left(t_{1}\right)-\beta_{2} p_{2}\left(t_{1}\right) x_{2}^{\nabla}\left(t_{1}\right)=\sum_{i=2}^{n-2} a_{i} x_{2}\left(\eta_{i}\right), \\
\gamma_{2} x_{2}\left(t_{n}\right)+\delta_{2} p_{1}\left(t_{n}\right) x_{2}^{\nabla}\left(t_{n}\right)=0, \\
\alpha_{1} x_{1}\left(t_{1}\right)-\beta_{1} p_{1}\left(t_{1}\right) x_{1}^{\nabla}\left(t_{1}\right)=0,
\end{gathered}
$$




$$
\begin{gathered}
\gamma_{1} x_{1}\left(t_{n}\right)+\delta_{1} p_{1}\left(t_{n}\right) x_{1}^{\nabla}\left(t_{n}\right)=\sum_{i=2}^{n-2} b_{i} x_{1}\left(\eta_{i}\right), \\
\alpha_{2} x_{2}\left(t_{1}\right)-\beta_{2} p_{2}\left(t_{1}\right) x_{2}^{\nabla}\left(t_{1}\right)=\sum_{i=2}^{n-2} a_{i} x_{2}\left(\eta_{i}\right), \\
\gamma_{2} x_{2}\left(t_{n}\right)+\delta_{2} p_{1}\left(t_{n}\right) x_{2}^{\nabla}\left(t_{n}\right)=0, \\
\alpha_{1} x_{1}\left(t_{1}\right)-\beta_{1} p_{1}\left(t_{1}\right) x_{1}^{\nabla}\left(t_{1}\right)=\sum_{i=2}^{n-2} a_{i} x_{1}\left(\eta_{i}\right), \\
\gamma_{1} x_{1}\left(t_{n}\right)+\delta_{1} p_{1}\left(t_{n}\right) x_{1}^{\nabla}\left(t_{n}\right)=0, \\
\alpha_{2} x_{2}\left(t_{1}\right)-\beta_{2} p_{2}\left(t_{1}\right) x_{2}^{\nabla}\left(t_{1}\right)=0, \\
\gamma_{2} x_{2}\left(t_{n}\right)+\delta_{2} p_{1}\left(t_{n}\right) x_{2}^{\nabla}\left(t_{n}\right)=\sum_{i=2}^{n-2} b_{i} x_{2}\left(\eta_{i}\right),
\end{gathered}
$$

where

$$
y(t)^{*}= \begin{cases}y(t), & y(t) \geq 0 \\ 0, & y(t)<0\end{cases}
$$

and $v_{i k}(t)=\lambda \int_{t_{1}}^{t_{n}} H_{i k}(t, s) g(s) \Delta s$. For $k=2, \ldots, 5$, from Lemma $1,\left(v_{1 k}(t), v_{2 k}(t)\right)$ is the solution of the equation

$$
\begin{array}{r}
\left(p_{1} v_{1}^{\nabla}\right)^{\Delta}(t)-q_{1}(t) v_{1}(t)+\lambda g(t)=0, \\
\lambda>0, t_{1}<t<t_{n}, \\
\left(p_{2} v_{2}^{\nabla}\right)^{\Delta}(t)-q_{2}(t) v_{2}(t)+\lambda g(t)=0,
\end{array}
$$$$
\lambda>0,
$$

respectively, satisfying the following boundary value conditions:

$$
\begin{gathered}
\alpha_{1} v_{1}\left(t_{1}\right)-\beta_{1} p_{1}\left(t_{1}\right) v_{1}^{\nabla}\left(t_{1}\right)=\sum_{i=2}^{n-2} a_{i} v_{1}\left(\eta_{i}\right), \\
\gamma_{1} v_{1}\left(t_{n}\right)+\delta_{1} p_{1}\left(t_{n}\right) v_{1}^{\nabla}\left(t_{n}\right)=0, \\
\alpha_{2} v_{2}\left(t_{1}\right)-\beta_{2} p_{2}\left(t_{1}\right) v_{2}^{\nabla}\left(t_{1}\right)=\sum_{i=2}^{n-2} a_{i} v_{2}\left(\eta_{i}\right), \\
\gamma_{2} v_{2}\left(t_{n}\right)+\delta_{2} p_{1}\left(t_{n}\right) v_{2}^{\nabla}\left(t_{n}\right)=0, \\
\alpha_{1} v_{1}\left(t_{1}\right)-\beta_{1} p_{1}\left(t_{1}\right) v_{1}^{\nabla}\left(t_{1}\right)=0, \\
\gamma_{1} v_{1}\left(t_{n}\right)+\delta_{1} p_{1}\left(t_{n}\right) v_{1}^{\nabla}\left(t_{n}\right)=\sum_{i=2}^{n-2} b_{i} v_{1}\left(\eta_{i}\right), \\
\alpha_{2} v_{2}\left(t_{1}\right)-\beta_{2} p_{2}\left(t_{1}\right) v_{2}^{\nabla}\left(t_{1}\right)=\sum_{i=2}^{n-2} a_{i} v_{2}\left(\eta_{i}\right), \\
\gamma_{2} v_{2}\left(t_{n}\right)+\delta_{2} p_{1}\left(t_{n}\right) v_{2}^{\nabla}\left(t_{n}\right)=0, \\
\alpha_{1} v_{1}\left(t_{1}\right)-\beta_{1} p_{1}\left(t_{1}\right) x_{1}^{\nabla}\left(t_{1}\right)=\sum_{i=2}^{n-2} a_{i} v_{1}\left(\eta_{i}\right), \\
\gamma_{1} v_{1}\left(t_{n}\right)+\delta_{1} p_{1}\left(t_{n}\right) x_{1}^{\nabla}\left(t_{n}\right)=0, \\
\alpha_{2} v_{2}\left(t_{1}\right)-\beta_{2} p_{2}\left(t_{1}\right) x_{2}^{\nabla}\left(t_{1}\right)=0, \\
\gamma_{2} v_{2}\left(t_{n}\right)+\delta_{2} p_{1}\left(t_{n}\right) x_{2}^{\nabla}\left(t_{n}\right)=\sum_{i=2}^{n-2} b_{i} v_{2}\left(\eta_{i}\right) .
\end{gathered}
$$

We will show that there exists a solution $\left(x_{1 k}, x_{2 k}\right)$ to the boundary value problem $(\bar{k})$ of the system (26) with $x_{i k}(t) \geq$ $v_{i k}(t), t \in\left[\rho\left(t_{1}\right), t_{n}\right]$. If this is true, then $u_{i k}(t)=x_{i k}(t)-v_{i k}(t)$ is a nonnegative solution (positive on $\left(\rho\left(t_{1}\right), t_{n}\right)$ ) of the system (1) with the boundary value problem $(k)$, (where $i=1,2 ; k=$ $2, \ldots, 5, \bar{k}=k+11)$. Since for any $t \in\left(t_{1}, t_{n}\right)$, from

$$
\begin{aligned}
\left(p_{i} x_{i k}^{\nabla}\right)^{\Delta}(t)-q_{i}(t) x_{i k}^{\nabla}(t) \\
\quad=\left(p_{i}\left(u_{i k}+v_{i k}\right)^{\nabla}\right)^{\Delta}(t)-q_{i}(t)\left(u_{i k}+v_{i k}\right)^{\nabla}(t) \\
\quad=-\lambda\left(f_{i}\left(t,\left[x_{1 k}(t)-v_{1 k}(t)\right]^{*},\left[x_{2 k}(t)-v_{2 k}(t)\right]^{*}\right)+g(t)\right) \\
=-\lambda\left(f_{i}\left(t, u_{1 k}(t), u_{2 k}(t)\right)+g(t)\right),
\end{aligned}
$$

we have

$$
\left(p_{i} u_{i k}^{\nabla}\right)^{\Delta}(t)-q_{i}(t) u_{i k}(t)=-\lambda f_{i}\left(t, u_{1 k}(t), u_{2 k}(t)\right) .
$$

As a result, we will concentrate our study on (26) with the boundary value problem $(\bar{k})$. 
Employing Lemma 1, we note that $\left(x_{1 k}(t), x_{2 k}(t)\right)$ is a solution of the system (26) with boundary value $(\bar{k})$ if and only if

$$
\begin{gathered}
x_{1 k}(t)=\lambda \int_{t_{1}}^{t_{n}} H_{1 k}(t, s)\left(f _ { 1 } \left(s,\left[x_{1 k}(s)-v_{1 k}(s)\right]^{*},\right.\right. \\
\left.\left[x_{2 k}(s)-v_{2 k}(s)\right]^{*}\right) \\
+g(s)) \Delta s, \\
t \in\left[\rho\left(t_{1}\right), t_{n}\right], \\
x_{2 k}(t)=\lambda \int_{t_{1}}^{t_{n}} H_{2 k}(t, s)\left(f _ { 2 } \left(s,\left[x_{1 k}(s)-v_{1 k}(s)\right]^{*},\right.\right. \\
\left.\left[x_{2 k}(s)-v_{2 k}(s)\right]^{*}\right) \\
+g(s)) \Delta s .
\end{gathered}
$$

We define a cone $P_{i j}(i, j=1,2)$ by

$$
P_{i j}=\left\{x \in E \mid x(t) \geq \frac{c_{*}}{C^{*}} \phi_{i j}(t)\|x\|, t \in\left[\rho\left(t_{1}\right), t_{n}\right]\right\} .
$$

It is clearly that $P_{i j} \times P_{m n}$ is a cone of $E \times E,(i, j, m, n=1,2)$. Define the integral operator $T_{2}: P_{11} \times P_{21} \rightarrow E \times E, T_{3}: P_{12} \times$ $P_{22} \rightarrow E \times E, T_{4}: P_{12} \times P_{21} \rightarrow E \times E, T_{5}: P_{21} \times P_{12} \rightarrow E \times E$, by

$$
\begin{array}{r}
T_{k}\left(x_{1 k}, x_{2 k}\right)=\left(T_{1 k}\left(x_{1 k}, x_{2 k}\right), T_{2 k}\left(x_{1 k}, x_{2 k}\right)\right), \\
(k=2, \ldots, 5),
\end{array}
$$

where operators $T_{i k}$ are defined by

$$
\begin{gathered}
T_{i k}\left(x_{1 k}, x_{2 k}\right)(t)=\lambda \int_{t_{1}}^{t_{n}} H_{i k}(t, s)\left(f _ { i } \left(s,\left[x_{1 k}(s)-v_{1 k}(s)\right]^{*},\right.\right. \\
\left.\left[x_{2 k}(s)-v_{2 k}(s)\right]^{*}\right) \\
+g(s)) \Delta s, \\
t \in\left[\rho\left(t_{1}\right), t_{n}\right],
\end{gathered}
$$

where $i=1,2$. Clearly, if $\left(x_{1 k}, x_{2 k}\right)$ is a fixed point of $T_{k}$, then $\left(x_{1 k}, x_{2 k}\right)$ is a solution of system (26) with $(\bar{k})(k=$ $2, \ldots, 5, \bar{k}=k+11)$.
For $k=2, \ldots, 5$, from (35) and Lemma 3, we have $T_{k}\left(x_{1 k}, x_{2 k}\right)(t) \geq 0$ on $[0,1]$, for $\left(x_{1 k}, x_{2 k}\right) \in P_{i j} \times P_{m n}$, we have

$$
\begin{aligned}
& T_{i k}\left(x_{1 k}, x_{2 k}\right)(t) \\
& =\lambda \int_{t_{1}}^{t_{n}} H_{i k}(t, s)\left(f _ { i } \left(s,\left[x_{1 k}(s)-v_{1 k}(s)\right]^{*},\right.\right. \\
& \left.\quad\left[x_{2 k}(s)-v_{2 k}(s)\right]^{*}\right) \\
& +g(s)) \Delta s \\
& \leq C^{*} \lambda \quad \\
& \times \int_{\rho\left(t_{1}\right)}^{\sigma\left(t_{n}\right)} G_{i}(s, s)\left(f_{i}\left(s,[x(s)-v(s)]^{*}\right)+g(s)\right) \Delta s,
\end{aligned}
$$

then $\left\|T_{i k}\left(x_{1 k}, x_{2 k}\right)\right\| \leq C^{*} \lambda \int_{\rho\left(t_{1}\right)}^{\sigma\left(t_{n}\right)} G_{i}(s, s)\left(f\left(t,[x(t)-v(t)]^{*}\right)+\right.$ $g(t)) \Delta s$.

On the other hand, when $k=2$, we have

$$
\begin{aligned}
& T_{i 2}\left(x_{1 k}, x_{2 k}\right)(t) \\
& =\lambda \int_{t_{1}}^{t_{n}} H_{i 2}(t, s)\left(f _ { i } \left(s,\left[x_{1 k}(s)-v_{1 k}(s)\right]^{*},\right.\right. \\
& \left.\left.\left[x_{2 k}(s)-v_{2 k}(s)\right]^{*}\right)+g(s)\right) \Delta s \\
& \geq \lambda \int_{t_{1}}^{t_{n}} c_{*} \phi_{i 1}(t) G_{i}(s, s)\left(f _ { i } \left(s,\left[x_{1 k}(s)-v_{1 k}(s)\right]^{*},\right.\right. \\
& \quad+g(s)) \Delta s \\
& \left.\left.\geq \frac{c_{*}}{C^{*}} \phi_{i 1}(t) \lambda \quad v_{2 k}(s)\right]^{*}\right) \\
& \quad \times \int_{\rho\left(t_{1}\right)}^{\sigma\left(t_{n}\right)} C^{*} G_{i}(s, s)\left(f_{i}\left(s,[x(s)-v(s)]^{*}\right)+g(s)\right) \Delta s \\
& \geq \frac{c_{*}}{C^{*}} \phi_{i 1}(t)\left\|T_{i 2}\left(x_{1 k}, x_{2 k}\right)\right\| .
\end{aligned}
$$

Thus, $T_{i 2}\left(P_{11} \times P_{21}\right) \subset P_{i 1}$. Hence $T_{2}\left(P_{11} \times P_{21}\right) \subset P_{11} \times P_{21}$. When $k=3$, we have

$$
\begin{array}{r}
T_{i 3}\left(x_{1 k}, x_{2 k}\right)(t) \\
=\lambda \int_{t_{1}}^{t_{n}} H_{i 3}(t, s)\left(f _ { i } \left(s,\left[x_{1 k}(s)-v_{1 k}(s)\right]^{*},\right.\right. \\
\left.\left[x_{2 k}(s)-v_{2 k}(s)\right]^{*}\right) \\
+g(s)) \Delta s
\end{array}
$$




$$
\begin{gathered}
\geq \lambda \int_{t_{1}}^{t_{n}} c_{*} \phi_{i 2}(t) G_{i}(s, s)\left(f _ { i } \left(s,\left[x_{1 k}(s)-v_{1 k}(s)\right]^{*},\right.\right. \\
\left.\left[x_{2 k}(s)-v_{2 k}(s)\right]^{*}\right) \\
+g(s)) \Delta s \\
\geq \frac{c_{*}}{C^{*}} \phi_{i 2}(t) \lambda \quad \\
\times \int_{\rho\left(t_{1}\right)}^{\sigma\left(t_{n}\right)} C^{*} G_{i}(s, s)\left(f_{i}\left(s,[x(s)-v(s)]^{*}\right)+g(s)\right) \Delta s \\
\geq \frac{c_{*}}{C^{*}} \phi_{i 2}(t)\left\|T_{i 3}\left(x_{1 k}, x_{2 k}\right)\right\| .
\end{gathered}
$$

Thus, $T_{i 3}\left(P_{12} \times P_{22}\right) \subset P_{i 2}$. Hence $T_{3}\left(P_{12} \times P_{22}\right) \subset P_{12} \times P_{22}$.

Similarly discussion, we also have $T_{4}\left(P_{12} \times P_{21}\right) \subset P_{12} \times P_{21}$, $T_{5}\left(P_{21} \times P_{12}\right) \subset P_{21} \times P_{12}$. In addition, standard arguments show that $T_{k}$ is a completely continuous operator.

For simplicity, we adopt the notation: $P_{14}=P_{25}:=P_{12}$ and $P_{24}=P_{15}:=P_{21}$, then, we can write $T_{k}\left(P_{1(k-1)} \times P_{2(k-1)}\right) \subset$ $P_{1(k-1)} \times P_{2(k-1)}$, that is, $T_{i k}\left(P_{1(k-1)} \times P_{2(k-1)}\right) \subset P_{i(k-1)},(i=$ $1,2, k=2, \ldots, 5)$.

Theorem 6. Suppose that $\left(H_{1}\right)-\left(H_{2}\right)$ hold. Then there exists a constant $\bar{\lambda}>0$ such that, for any $0<\lambda \leq \bar{\lambda}$, (1) with boundary value condition $(k)$ has at least one positive solution $(k=2, \ldots, 5)$.

Proof. Fix $\delta \in(0,1)$ and $k(k=2, \ldots, 5)$. From $\left(\mathrm{H}_{2}\right)$ let $0<$ $\varepsilon<1$ be such that

$f_{i}\left(t, z_{1}, z_{2}\right) \geq \delta f_{i}(t, 0,0), \quad$ for $t_{1} \leq t \leq t_{n}, \quad 0 \leq z_{i} \leq \varepsilon, i=1,2$.

Let $\bar{f}(\varepsilon)=\max _{t_{1} \leq t \leq t_{n}, 0 \leq z_{1}, z_{2} \leq \varepsilon}\left\{\max _{i=1,2}\left\{f_{i}\left(t, z_{1}, z_{2}\right)\right\}+\right.$ $g(t)\}$, and $c=\int_{t_{1}}^{t_{n}} C^{*} G_{i}(s, s) \Delta s$. We have

$$
\lim _{z \downarrow 0} \frac{\bar{f}(z)}{z}=+\infty .
$$

Set $\bar{\lambda}=\varepsilon / 4 c \bar{f}(\varepsilon)$, since for any $0<\lambda \leq \bar{\lambda}$, fix the $\lambda \epsilon$ $(0, \bar{\lambda}]$, we always have

$$
\begin{gathered}
\lim _{z \downarrow 0} \frac{\bar{f}(z)}{z}=+\infty, \\
\frac{\bar{f}(\varepsilon)}{\varepsilon}<\frac{1}{4 c \lambda},
\end{gathered}
$$

Then there exists a $R_{0} \in(0, \varepsilon]$ such that

$$
\frac{\bar{f}\left(R_{0}\right)}{R_{0}}=\frac{1}{4 c \lambda} \text {. }
$$

Let $U_{k}=\left\{\left(x_{1 k}, x_{2 k}\right) \in P_{1(k-1)} \times P_{2(k-1)}:\left\|\left(x_{1 k}, x_{2 k}\right)\right\|_{1}<\right.$ $\left.R_{0}\right\},\left(x_{1 k}, x_{2 k}\right) \in \partial U_{k}$ and $\nu \in(0,1)$ be such that $\left(x_{1 k}, x_{2 k}\right)=$ $v T_{k}\left(x_{1 k}, x_{2 k}\right)$, that is, $x_{i k}=v T_{i k}\left(x_{1 k}, x_{2 k}\right)(i=1,2)$. We claim that $\left\|\left(x_{1 k}, x_{2 k}\right)\right\|_{1} \neq R_{0}$. In fact for $\left(x_{1 k}, x_{2 k}\right) \in \partial U_{k}$ and $\left\|\left(x_{1 k}, x_{2 k}\right)\right\|_{1}=R_{0}$, we have

$$
\begin{aligned}
& x_{i k}= v T_{i k}\left(x_{1 k}, x_{2 k}\right) \\
& \leq \lambda \int_{t_{1}}^{t_{n}} H_{i k}(t, s)\left(f _ { i } \left(s,\left[x_{1 k}(s)-v_{1 k}(s)\right]^{*},\right.\right. \\
&\left.\left.\quad\left[x_{2 k}(s)-v_{2 k}(s)\right]^{*}\right)+g(s)\right) \Delta s \\
& \leq \lambda \int_{t_{1}}^{t_{n}} C^{*} G_{i}(s, s)\left(f _ { i } \left(s,\left[x_{1 k}(s)-v_{1 k}(s)\right]^{*},\right.\right. \\
&\left.\left.\quad\left[x_{2 k}(s)-v_{2 k}(s)\right]^{*}\right)+g(s)\right) \Delta s \\
& \leq \lambda \int_{t_{1}}^{t_{n}} C^{*} G_{i}(s, s) \bar{f}\left(R_{0}\right) \Delta s \\
& \leq \lambda c \bar{f}\left(R_{0}\right) .
\end{aligned}
$$

It follows that

$$
R_{0}=\left\|\left(x_{1 k}, x_{2 k}\right)\right\|_{1} \leq 2 \lambda c \bar{f}\left(R_{0}\right),
$$

that is,

$$
\frac{\bar{f}\left(R_{0}\right)}{R_{0}} \geq \frac{1}{2 c \lambda}>\frac{1}{4 c \lambda}=\frac{\bar{f}\left(R_{0}\right)}{R_{0}},
$$

which implies that $\left\|\left(x_{1 k}, x_{2 k}\right)\right\|_{1} \neq R_{0}$. By the nonlinear alternative of Leray-Schauder type, $T_{k}$ has a fixed point $\left(x_{i 1}, x_{i 2}\right) \epsilon$ $\overline{U_{k}}$. Moreover combining $(40)$ and the fact that $R_{0}<\varepsilon$, we obtain

$$
\begin{aligned}
x_{i k}=\lambda \int_{t_{1}}^{t_{n}} H_{i k}(t, s)\left(f _ { i } \left(t,\left[x_{1 k}(t)-v_{1 k}(t)\right]^{*},\right.\right. \\
\left.\left.\quad\left[x_{2 k}(t)-v_{2 k}(t)\right]^{*}\right)+g(t)\right) \Delta s \\
\geq \lambda \int_{t_{1}}^{t_{n}} H_{i k}(t, s)(\delta f(s, 0,0)+g(t)) \Delta s \\
\geq \lambda \int_{t_{1}}^{t_{n}} H_{i k}(t, s) g(t) \Delta s \\
=v_{i k}(t) \quad \text { for } t \in\left(\rho\left(t_{1}\right), t_{n}\right) .
\end{aligned}
$$

Then $T_{k}$ has a positive fixed point $\left(x_{i 1}, x_{i 2}\right)$ and $\left\|\left(x_{i 1}, x_{i 2}\right)\right\|_{1} \leq R_{0}<1$; that is, $\left(x_{i 1}, x_{i 2}\right)$ is a positive solution of the boundary value problem (26) with $x_{i k}>v_{i k}(t)$ for $t \in\left(t_{1}, t_{n}\right)$.

Let $u_{i k}(t)=x_{i k}(t)-v_{i k}(t) \geq 0(i=1,2)$, then $\left(u_{1 k}, u_{2 k}\right)$ is a nonnegative solution (positive on $\left.\left(\rho\left(t_{1}\right), t_{n}\right)\right)$ of the boundary value problem (1).

Theorem 7. Suppose that $\left(H_{1}^{*}\right)$ and $\left(H_{3}\right)-\left(H_{4}\right)$ hold. Then there exists a constant $\lambda^{*}>0$ such that, for any $0<\lambda \leq \lambda^{*}$, (1) with boundary value condition $(k)$ has at least one positive solution $(k=2, \ldots, 5)$. 
Proof. We fix $k(k=2, \ldots, 5)$. Let $\Omega_{1}=\left\{\left(x_{1 k}, x_{2 k}\right) \in E \times\right.$ $\left.E:\left\|x_{i k}\right\|<R_{1}, i=1,2\right\}$, where $R_{1}=\max \{1, r\}$ and $r=$ $\left(C^{* 2} / c_{*}\right) \int_{t_{1}}^{t_{n}} g(s) \Delta s$. Choose

$$
\lambda^{*}=\min \left\{1, \frac{R_{1}}{2}(R+1)^{-1}, \frac{R_{1}}{2 r}\right\}
$$

where $R=\int_{t_{1}}^{t_{n}} C^{*} G_{i}(s, s)\left(\max _{0 \leq z_{1}, z_{2} \leq R_{1}} f_{i}\left(s, z_{1}, z_{2}\right)+g(s)\right) \Delta s$ and $R \geq 0$.

Then for any $\left(x_{1 k}, x_{2 k}\right) \in\left(P_{1(k-1)} \times P_{2(k-1)}\right) \cap \partial \Omega_{1}, x_{i k}(s)-$ $v_{i k}(s) \leq x_{i k}(s) \leq\left\|x_{i k}\right\| \leq R_{1}(i=1,2)$ and for $0<\lambda \leq \lambda^{*}$, we have

$$
\begin{aligned}
& \left\|T_{i k}\left(x_{1 k}, x_{2 k}\right)(t)\right\| \\
& \leq \lambda \int_{t_{1}}^{t_{n}} C^{*} G_{i}(s, s)\left(f _ { i } \left(s,\left[x_{1 k}(s)-v_{1 k}(s)\right]^{*},\right.\right. \\
& \left.\left.\quad\left[x_{2 k}(s)-v_{2 k}(s)\right]^{*}\right)+g(s)\right) \Delta s \\
& \leq \lambda \int_{t_{1}}^{t_{n}} C^{*} G_{i}(s, s)\left(\max _{0 \leq z_{1}, z_{2} \leq R_{1}} f_{i}\left(s, z_{1}, z_{2}\right)+g(s)\right) \Delta s \\
& \leq \lambda R \\
& \leq \frac{R_{1}}{2} .
\end{aligned}
$$

This implies

$$
\begin{aligned}
& \left\|T_{k}\left(x_{1 k}, x_{2 k}\right)\right\|_{1} \leq R_{1} \leq\left\|\left(x_{1 k}, x_{2 k}\right)\right\|_{1}, \\
& \left(x_{1 k}, x_{2 k}\right) \in\left(P_{1(k-1)} \times P_{2(k-1)}\right) \cap \partial \Omega_{1} .
\end{aligned}
$$

Choose a constant $N>1$ such that

$$
\lambda N \gamma \frac{c_{*}}{2\left(\left\|\phi_{i 1}\right\|+\left\|\phi_{i 2}\right\|\right)} \int_{\theta_{1}}^{\theta_{2}} G_{i}(s, s) \phi_{i 1}(s) \phi_{i 2}(s) \Delta s \geq 1,
$$

where $\gamma=\min _{k} \min _{\theta_{1} \leq t \leq \theta_{2}}\left\{\phi_{1 k}(t), \phi_{2 k}(t)\right\}$.

By assumption $\left(\mathrm{H}_{3}\right)$ and $\left(\mathrm{H}_{4}\right)$, there exists a constant $B>$ $R_{1}$ such that

$$
\begin{gathered}
\frac{f_{i}\left(t, z_{1}, z_{2}\right)}{z_{1}+z_{2}}>N, \text { that is, } f_{i}\left(t, z_{1}, z_{2}\right)>N\left(z_{1}+z_{2}\right), \\
\text { for } t \in\left[\theta_{1}, \theta_{2}\right], z_{1}+z_{2}>B(i=1,2) .
\end{gathered}
$$

Choose $R_{2}=\max \left\{R_{1}+1,2 \lambda r, 2 C^{*}(B+1) / c_{*} \gamma\right\}$ and let $\Omega_{2}=\left\{\left(x_{1 k}, x_{2 k}\right) \in E \times E:\left\|x_{i k}\right\|<R_{2}, i=1,2\right\}$. We note that $x(t) \geq\left(c_{*} / C^{*}\right) \phi_{i j}(t)\|x\|$ for all $x \in P_{i j}$, by Lemma 3, we have $H_{i k}(t, s) \leq\left(C^{* 2} / c_{*}\right)(x(t) /\|x\|)$. Then for any $\left(x_{1 k}, x_{2 k}\right) \in$
$\left(P_{1(k-1)} \times P_{2(k-1)}\right) \cap \partial \Omega_{2}$, we have $\left\|x_{1 k}\right\|=R_{2}$ or $\left\|x_{2 k}\right\|=R_{2}$. Without loss of generality let $\left\|x_{1 k}\right\|=R_{2}$, so we have

$$
\begin{aligned}
x_{1 k}(t)-v_{1 k}(t) & =x_{1 k}(t)-\lambda \int_{t_{1}}^{t_{n}} H_{1 k}(t, s) g(s) \Delta s \\
& \geq x_{1 k}(t)-\lambda \int_{t_{1}}^{t_{n}} \frac{C^{* 2}}{c_{*}} \frac{x_{1 k}(t)}{\left\|x_{1 k}\right\|} g(s) \Delta s \\
& =x_{1 k}(t)-\lambda \frac{x_{1 k}(t)}{\left\|x_{1 k}\right\|} \int_{t_{1}}^{t_{n}} \frac{C^{* 2}}{c_{*}} g(s) \Delta s \\
& \geq x_{1 k}(t)-\frac{x_{1 k}(t)}{\left\|x_{1 k}\right\|} \lambda r \\
& \geq x_{1 k}(t)-\frac{x_{1 k}(t)}{R_{2}} \lambda r \\
& \geq\left(1-\frac{\lambda r}{R_{2}}\right) x_{1 k}(t) \\
& \geq \frac{1}{2} x_{1 k}(t) \geq 0, \quad t \in\left[\rho\left(t_{1}\right), t_{n}\right] .
\end{aligned}
$$

Thus

$$
\begin{gathered}
\min _{\theta_{1} \leq t \leq \theta_{2}}\left\{\left[x_{1 k}(t)-v_{1 k}(t)\right]^{*}+\left[x_{2 k}(t)-v_{2 k}(t)\right]^{*}\right\} \\
\geq \min _{\theta_{1} \leq t \leq \theta_{2}}\left\{x_{1 k}(t)-v_{1 k}(t)\right\} \geq \min _{\theta_{1} \leq t \leq \theta_{2}}\left\{\frac{1}{2} x_{1 k}(t)\right\} \\
\geq \min _{\theta_{1} \leq t \leq \theta_{2}}\left\{\frac{c_{*}}{2 C^{*}} \phi_{1 k}(t)\left\|x_{1 k}\right\|, \frac{c_{*}}{2 C^{*}} \phi_{2 k}(t)\left\|x_{1 k}\right\|\right\} \\
=\frac{c_{*}}{2 C^{*}} R_{2} \min _{\theta_{1} \leq t \leq \theta_{2}}\left\{\phi_{1 k}(t), \phi_{2 k}(t)\right\} \geq B+1>B .
\end{gathered}
$$

Now since $B>R_{1}$, it follows that

$$
\begin{gathered}
T_{i k}\left(x_{1 k}, x_{2 k}\right)(t) \\
=\lambda \int_{t_{1}}^{t_{n}} H_{i k}(t, s)\left(f _ { i } \left(s,\left[x_{1 k}(s)-v_{1 k}(s)\right]^{*},\right.\right. \\
\left.\left.\left[x_{2 k}(s)-v_{2 k}(s)\right]^{*}\right)+g(s)\right) \Delta s \\
\geq \lambda \int_{\theta_{1}}^{\theta_{2}} H_{i k}(t, s)\left(f _ { i } \left(s,\left[x_{1 k}(s)-v_{1 k}(s)\right]^{*},\right.\right. \\
\left.\left.\left[x_{2 k}(s)-v_{2 k}(s)\right]^{*}\right)+g(s)\right) \Delta s \\
\geq \lambda \int_{\theta_{1}}^{\theta_{2}} H_{i k}(t, s) f_{i}\left(s,\left[x_{1 k}(s)-v_{1 k}(s)\right]^{*},\right. \\
\left.\left[x_{2 k}(s)-v_{2 k}(s)\right]^{*}\right) \Delta s
\end{gathered}
$$




$$
\begin{aligned}
& \geq \lambda \int_{\theta_{1}}^{\theta_{2}} H_{i k}(t, s) N\left(\left[x_{1 k}(s)-v_{1 k}(s)\right]^{*}\right. \\
& \left.+\left[x_{2 k}(s)-v_{2 k}(s)\right]^{*}\right) \Delta s \\
& \geq \lambda \int_{\theta_{1}}^{\theta_{2}} H_{i k}(t, s) N\left(x_{1 k}(s)-v_{1 k}(s)\right) \Delta s \\
& \geq \lambda \int_{\theta_{1}}^{\theta_{2}} c_{*} \min _{\theta_{1} \leq t \leq \theta_{2}}\left\{\phi_{1 k}(t), \phi_{2 k}(t)\right\} G_{i}(s, s) N\left(x_{1 k}(s)-v_{1 k}(s)\right) \Delta s \\
& \geq \lambda \int_{\theta_{1}}^{\theta_{2}} c_{*} \min _{\theta_{1} \leq t \leq \theta_{2}}\left\{\phi_{1 k}(t), \phi_{2 k}(t)\right\} G_{i}(s, s) \frac{N}{2} x_{1 k}(s) d s \\
& \geq \lambda \min _{\theta_{1} \leq t \leq \theta_{2}}\left\{\phi_{1 k}(t), \phi_{2 k}(t)\right\} \\
& \times \int_{\theta_{1}}^{\theta_{2}} c_{*} G_{i}(s, s) \frac{c_{*} N}{2\left(\left\|\phi_{i 1}\right\|+\left\|\phi_{i 2}\right\|\right)} \phi_{i 1}(s) \phi_{i 2}(s)\left\|x_{1 k}\right\| \Delta s \\
& \geq \lambda N \gamma \frac{c_{*}}{2\left(\left\|\phi_{i 1}\right\|+\left\|\phi_{i 2}\right\|\right)} \int_{\theta_{1}}^{\theta_{2}} G_{i}(s, s) \phi_{i 1}(s) \phi_{i 2}(s) \Delta s R_{2} \\
& \geq R_{2}, \quad t \in\left[\theta_{1}, \theta_{2}\right] \text {. }
\end{aligned}
$$

This implies

$$
\begin{gathered}
\left\|T_{k}\left(x_{1 k}, x_{2 k}\right)\right\|_{1} \geq\left\|\left(x_{1 k}, x_{2 k}\right)\right\|_{1}, \\
\left(x_{1 k}, x_{2 k}\right) \in\left(P_{1(k-1)} \times P_{2(k-1)}\right) \cap \partial \Omega_{2} .
\end{gathered}
$$

For the Krasnosel'skii's fixed point theorem, one deduces that $T_{k}$ has a fixed point $\left(x_{1 k}, x_{2 k}\right)$ with $R_{1}<\left\|\left(x_{1 k}, x_{2 k}\right)\right\|<R_{2} \Leftrightarrow$ $R_{1}<\left\|x_{1 k}\right\|+\left\|x_{2 k}\right\|<R_{2}$.

Since $r \leq R_{1}<\left\|x_{i k}\right\|<R_{2}(i=1,2)$, then

$$
\begin{aligned}
x_{i k}(t)-v_{i k}(t) & =x_{i k}(t)-\lambda \int_{t_{1}}^{t_{n}} H_{i k}(t, s) g(s) \Delta s \\
& \geq x_{i k}(t)-\lambda \int_{t_{1}}^{t_{n}} \frac{C^{* 2}}{c_{*}} \frac{x_{i k}(t)}{\left\|x_{i k}\right\|} g(s) \Delta s \\
& =x_{i k}(t)-\lambda \frac{x_{i k}(t)}{\left\|x_{i k}\right\|} r \\
& \geq x_{i k}(t)-\lambda x_{i k}(t) \\
& =(1-\lambda) x_{i k}(t) \\
& \geq(1-\lambda) \frac{c_{*}}{C^{*}} \frac{\phi_{i 1}(t) \phi_{i 2}(t)}{\left\|\phi_{i 1}\right\|+\left\|\phi_{i 2}\right\|}\left\|x_{i k}\right\| \\
& >0, \quad t \in\left(\rho\left(t_{1}\right), t_{n}\right) .
\end{aligned}
$$

Thus $\left(x_{1 k}, x_{2 k}\right)$ is a positive solution of the boundary value problem (26) with $x_{i k}(t)>v_{i k}(t)(i=1,2)$ for $t \in\left(\rho\left(t_{1}\right), t_{n}\right)$.

Let $u_{i k}(t)=x_{i k}(t)-v_{i k}(t) \geq 0(i=1,2)$, then $\left(u_{1 k}, u_{2 k}\right)$ is a nonnegative solution (positive on $\left.\left(\rho\left(t_{1}\right), t_{n}\right)\right)$ of the boundary value problem (1).

Since condition $\left(\mathrm{H}_{1}\right)$ implies conditions $\left(\mathrm{H}_{1}^{*}\right)$ and $\left(\mathrm{H}_{4}\right)$ then from the proof of Theorems 6 and 7, we immediately have the following theorem.
Theorem 8. Suppose that $\left(H_{1}\right)-\left(H_{3}\right)$ hold. Then (1) with boundary value condition $(k)$ has at least two positive solutions for $\lambda>0$ sufficiently small $(k=2, \ldots, 5)$.

In fact with $0<\lambda<\min \left\{\bar{\lambda}, \lambda^{*}\right\}$ then (1) with boundary value condition $(k)$ has at least two positive solutions.

Remark 9. In Theorems 6-8, we use the assumption condition 16. If we have not the condition 16, that is, $a_{i}=b_{i}=0$, then the system (1) and boundary condition $(k)$ are

$$
\begin{gathered}
\left(p_{1} u_{1}^{\nabla}\right)^{\Delta}(t)-q_{1}(t) u_{1}(t)+\lambda f_{1}\left(t, u_{1}(t), u_{2}(t)\right)=0, \\
t \in\left(t_{1}, t_{n}\right), \lambda>0, \\
\left(p_{2} u_{2}^{\nabla}\right)^{\Delta}(t)-q_{2}(t) u_{2}(t)+\lambda f_{2}\left(t, u_{1}(t), u_{2}(t)\right)=0, \\
\alpha_{1} u_{1}\left(t_{1}\right)-\beta_{1} p_{1}\left(t_{1}\right) u_{1}^{\nabla}\left(t_{1}\right)=0, \\
\gamma_{1} u_{1}\left(t_{n}\right)+\delta_{1} p_{1}\left(t_{n}\right) u_{1}^{\nabla}\left(t_{n}\right)=0, \\
\alpha_{2} u_{2}\left(t_{1}\right)-\beta_{2} p_{2}\left(t_{1}\right) u_{2}^{\nabla}\left(t_{1}\right)=0, \\
\gamma_{2} u_{2}\left(t_{n}\right)+\delta_{2} p_{1}\left(t_{n}\right) u_{2}^{\nabla}\left(t_{n}\right)=0 .
\end{gathered}
$$

From Lemma 2, an argument similar to those in Theorems 6-8 yields the following theorems.

Theorem 10. Suppose that $\left(H_{1}\right)$ and $\left(H_{2}\right)$ hold. Then there exists a constant $\bar{\lambda}>0$ such that, for any $0<\lambda \leq \bar{\lambda}$, the boundary value problem (58) has at least one positive solution.

Theorem 11. Suppose that $\left(H_{1}^{*}\right)$ and $\left(H_{3}\right)-\left(H_{4}\right)$ hold. Then there exists a constant $\lambda^{*}>0$ such that, for any $0<\lambda \leq \lambda^{*}$, the boundary value problem (58) has at least one positive solution.

Theorem 12. Suppose that $\left(H_{1}\right)-\left(H_{4}\right)$ hold. Then the boundary value problem (58) has at least two positive solutions for $\lambda>0$ sufficiently small.

\section{Example}

To illustrate the usefulness of the results, we give some examples.

Example 13. Consider the boundary value problem

$$
\begin{gathered}
u^{\prime \prime}-u=-\lambda\left((u+v)^{a}+\frac{1}{\left(t-t^{2}\right)^{1 / 2}} \cos (2 \pi(u+v))\right), \\
-1<t<1, \lambda>0, \\
v^{\prime \prime}-v=-\lambda\left((u-1)^{2}+v^{2}+\frac{1}{\left(t-t^{2}\right)^{1 / 2}} \sin (2 \pi u)\right), \\
u(-1)=v(1)=0, \quad u(1)=a u(0), \quad v(-1)=b v(0),
\end{gathered}
$$

where $a>1$. Then if $\lambda>0$ is sufficiently small, (59) has a positive solution $u$ with $u(t)>0$ for $t \in(0,1)$. 
To see this, we will apply Theorem 7 with

$$
\begin{gathered}
f_{1}(t, u, v)=(u+v)^{a}+\frac{1}{\left(t^{2}-t^{4}\right)^{1 / 4}} \cos (2 \pi(u+v)), \\
f_{2}(t, u, v)=(u-1)^{2}+v^{2}+\frac{1}{\left(t^{2}-t^{4}\right)^{1 / 4}} \sin (2 \pi u), \\
g_{1}(t)=g_{2}(t)=g(t)=\frac{1}{\left(t^{2}-t^{4}\right)^{1 / 4}} .
\end{gathered}
$$

Clearly for $t \in(0,1)$,

$$
\begin{gathered}
f_{i}(t, u, v)+g(t)>0, \quad \text { for } t \in(0,1) i=1,2, \\
\lim _{u+v \uparrow+\infty} \inf \frac{f_{i}(t, u, v)}{u+v}=+\infty \quad \text { for all } t \in\left[\theta_{1}, \theta_{2}\right] \subset(0,1) .
\end{gathered}
$$

Now $\left(\mathrm{H}_{1}^{*}\right),\left(\mathrm{H}_{3}\right)$, and $\left(\mathrm{H}_{4}\right)$ hold. We note that the boundary condition of (59) is in accord with (4), and from [1], we have

$$
\begin{gathered}
\phi_{11}=\phi_{21}=\frac{e^{t+1}-e^{-t-1}}{2}, \quad \phi_{12}=\phi_{22}=\frac{e^{-t+1}-e^{t-1}}{2}, \\
d_{1}=d_{2}=\sinh (2) .
\end{gathered}
$$

Then

$$
\begin{aligned}
G_{1}(t, s) & =G_{2}(t, s) \\
& =\frac{1}{d_{1}} \begin{cases}\phi_{12}(t) \phi_{11}(s), & \rho\left(t_{1}\right) \leq s \leq t \leq t_{n}, \\
\phi_{11}(t) \phi_{12}(s), & \rho\left(t_{1}\right) \leq t \leq s \leq t_{n},\end{cases} \\
H_{14}(t, s) & =G_{1}(t, s)+\frac{1}{d_{1}-a \phi_{11}(0)} a G_{1}(0, s) \phi_{11}(t), \\
H_{24}(t, s) & =G_{2}(t, s)+\frac{1}{d_{2}-b \phi_{22}(0)} b G_{2}(0, s) \phi_{22}(t) .
\end{aligned}
$$

Note $r=\int_{t_{1}}^{t_{n}}\left(C^{* 2} / c_{*}\right) g(s) \Delta s$. Let $R_{1}=r+1$ and we have

$$
\begin{aligned}
R & =\int_{t_{1}}^{t_{n}} C^{*} G_{i}(s, s)\left(\max _{0 \leq z_{1}, z_{2} \leq R_{1}} f_{i}\left(s, z_{1}, z_{2}\right)+g(s)\right) \Delta s \\
& \leq \int_{t_{1}}^{t_{n}} C^{*} G_{i}(s, s)\left[2^{a+2} R_{1}^{a+2}+\frac{2}{\left(s^{2}-s^{4}\right)^{1 / 4}}\right] \Delta s \\
& \leq \int_{-1}^{1} \frac{C^{*} e^{4}}{4}\left[2^{a+2} R_{1}^{a+2}+\frac{2}{\left(s^{2}-s^{4}\right)^{1 / 4}}\right] \Delta s \\
& =\int_{0}^{1} \frac{C^{*} e^{4}}{2}\left[2^{a+2} R_{1}^{a+2}+\frac{2}{\left(s^{2}-s^{4}\right)^{1 / 4}}\right] \Delta s \\
& \leq \int_{0}^{1} \frac{C^{*} e^{4}}{2}\left[2^{a+2} R_{1}^{a+2}+\frac{2}{\left(s-s^{2}\right)^{1 / 2}}\right] \Delta s \\
& \leq 2^{a+1} C^{*} e^{4}\left(R_{1}^{a+2}+\pi\right) .
\end{aligned}
$$

Also let

$$
\lambda^{*}=\min \left\{1, \frac{R_{1}}{2^{a+2} C^{*} e^{4}}\left(R_{1}^{a+2}+\pi\right)^{-1}, \frac{R_{1}}{2 r}\right\} .
$$

Now, if $\lambda<\lambda^{*}$, Theorem 7 guarantees that (59) has a positive solutions $(u, v)$ with $\|u\| \geq 1$ and $\|v\| \geq 1$.

Example 14. Consider the boundary value problem:

$$
\begin{gathered}
\left(p_{1} u_{1}^{\nabla}\right)^{\Delta}(t)-q_{1}(t) u_{1}(t) \\
=-\lambda\left(e^{u_{1}}+u_{2}^{2}+7 \cos \left(2 \pi t u_{1}\right)\right), \\
t_{1}<t<t_{n}, \quad \lambda>0, \\
\left(p_{2} u_{2}^{\nabla}\right)^{\Delta}(t)-q_{2}(t) u_{2}(t) \\
=-\lambda\left(\left(u_{1}-1\right)^{2}+u_{2}^{2}+5 \sin \left(2 \pi t u_{2}\right)\right)
\end{gathered}
$$

satisfying one of the boundary value conditions $(k),(k=$ $2, \ldots, 5)$.

Then if $\lambda>0$ is sufficiently small, (66) has two solutions $\left(u_{11}, u_{12}\right),\left(u_{21}, u_{22}\right)$ with $u_{i j}(t)>0$ for $t \in(0,1), i, j=1,2$.

To see this, we will apply Theorem 8 with

$$
\begin{gathered}
f_{1}\left(t, u_{1}, u_{2}\right)=e^{u_{1}}+u_{2}^{2}+7 \cos \left(2 \pi t u_{1}\right), \\
f_{2}\left(t, u_{1}, u_{2}\right)=\left(u_{1}-1\right)^{2}+u_{2}^{2}+5 \sin \left(2 \pi t u_{2}\right), \\
g_{1}(t)=g_{2}(t)=g(t)=8 .
\end{gathered}
$$

Clearly, for $t \in(0,1)$,

$$
\begin{gathered}
f_{i}\left(t, u_{1}, u_{2}\right)+g(t) \geq 1>0, \\
f_{1}(t, 0,0)=8>0, \\
f_{2}(t, 0,0)=3>0, \\
\lim _{u+v \uparrow+\infty} \inf \frac{f_{i}\left(t, u_{1}, u_{2}\right)}{u_{1}+u_{2}}=+\infty, \quad i=1,2 .
\end{gathered}
$$

Now $\left(\mathrm{H}_{1}\right)-\left(\mathrm{H}_{4}\right)$ hold. Let $\delta=1 / 100, \varepsilon=1 / 8$, and we have $f_{i}\left(t, u_{1}, u_{2}\right) \geq \delta f_{i}(t, 0,0), \quad$ for $0 \leq t \leq 1,0 \leq u_{i} \leq \varepsilon, i=1,2$.

Furthermore let $\quad \bar{f}(\varepsilon)$ = $\max _{0 \leq t \leq 1,0 \leq u_{1}, u_{2} \leq \varepsilon}\left\{\max _{i=1,2} f_{i}\left(t, u_{1}, u_{2}\right)+g(t)\right\}$, and $c=$ $\int_{t_{1}}^{t_{n}} C^{*} G_{i}(s, s) \Delta s$. Note

$$
\frac{\varepsilon}{4 c \bar{f}(\varepsilon)} \geq \frac{1}{32 c(e+8)}>\frac{1}{352 c} .
$$

Let $\bar{\lambda}=1 / 352 c$. Now, if $0<\lambda<\bar{\lambda}$ then $0<\lambda<\varepsilon / 4 c \bar{f}(\varepsilon)$ and Theorem 6 guarantees that (66) has positive solutions $\left(u_{11}, u_{12}\right)$ with $\left\|u_{1 j}\right\| \leq(1 / 8)(j=1,2)$. 

have

Next note $r=8 C^{* 2}\left(t_{n}-t_{1}\right) / c_{*}$ and let $R_{1}=r+2$ so we

$$
\begin{aligned}
R & =\int_{0}^{1} C^{*} G_{i}(s, s)\left(\max _{0 \leq z_{1}, z_{2} \leq R_{1}} f_{i}\left(s, z_{1}, z_{2}\right)+g(s)\right) \Delta s \\
& \leq \int_{0}^{1} C^{*} G_{i}(s, s)\left(e^{R_{1}}+2 R_{1}^{2}+7+8\right) \Delta s \\
& \leq \int_{0}^{1} C^{*} G_{i}(s, s) \Delta s\left(e^{R_{1}}+2 R_{1}^{2}+15\right) \\
& \leq\left(e^{R_{1}}+2 R_{1}^{2}+15\right) c .
\end{aligned}
$$

Also let

$$
\lambda^{*}=\min \left\{1, \frac{R_{1}}{2\left(e^{R_{1}}+2 R_{1}^{2}+15\right) c}, \frac{R_{1}}{2 r}\right\} .
$$

Now, if $\lambda<\lambda^{*}$, Theorem 7 guarantees that (59) has a positive solutions $\left(u_{21}, u_{22}\right)$ with $\left\|u_{2 j}\right\| \geq 2, j=1,2$.

Thus, if $\lambda<\min \left\{\bar{\lambda}, \lambda^{*}\right\}$, Theorem 8 guarantees that (66) has two solutions $\left(u_{11}, u_{12}\right)$ and $\left(u_{21}, u_{22}\right)$ with $u_{i j}>0$ for $t \in$ $(0,1), i, j=1,2$.

\section{References}

[1] D. R. Anderson and R. Ma, "Second-order n-point eigenvalue problems on time scales," Advances in Difference Equations, vol. 2006, Article ID 59572, 17 pages, 2006.

[2] M. Feng, X. Zhang, and W. Ge, "Multiple positive solutions for a class of $m$-point boundary value problems on time scales," Advances in Difference Equations, vol. 2009, Article ID 219251, 14 pages, 2009.

[3] S. G. Topal and A. Yantir, "Positive solutions of a second order $m$-point BVP on time scales," Nonlinear Dynamics and Systems Theory, vol. 9, no. 2, pp. 185-197, 2009.

[4] C. Yuan and Y. Liu, "Multiple positive solutions of a second order nonlinear semipositone $m$-point boundary value problem on time scales," Abstract and Applied Analysis, vol. 2010, Article ID 261741, 19 pages, 2010.

[5] X. Lin and Z. Du, "Positive solutions of $m$-point boundary value problem for second-order dynamic equations on time scales," Journal of Difference Equations and Applications, vol. 14, no. 8, pp. 851-864, 2008.

[6] H.-R. Sun and W.-T. Li, "Positive solutions for nonlinear threepoint boundary value problems on time scales," Journal of Mathematical Analysis and Applications, vol. 299, no. 2, pp. 508524, 2004.

[7] Y. Pang and Z. Bai, "Upper and lower solution method for a fourth-order four-point boundary value problem on time scales," Applied Mathematics and Computation, vol. 215, no. 6, pp. 2243-2247, 2009.

[8] F. M. Atici and S. G. Topal, "The generalized quasilinearization method and three point boundary value problems on time scales," Applied Mathematics Letters, vol. 18, no. 5, pp. 577-585, 2005.

[9] S. Liang and J. Zhang, "The existence of countably many positive solutions for nonlinear singular $m$-point boundary value problems on time scales," Journal of Computational and Applied Mathematics, vol. 223, no. 1, pp. 291-303, 2009.
[10] S. Liang, J. Zhang, and Z. Wang, "The existence of three positive solutions of $m$-point boundary value problems for some dynamic equations on time scales," Mathematical and Computer Modelling, vol. 49, no. 7-8, pp. 1386-1393, 2009.

[11] L. Hu, "Positive solutions to singular third-order three-point boundary value problems on time scales," Mathematical and Computer Modelling, vol. 51, no. 5-6, pp. 606-615, 2010.

[12] I. Yaslan, "Multiple positive solutions for nonlinear threepoint boundary value problems on time scales," Computers \& Mathematics with Applications, vol. 55, no. 8, pp. 1861-1869, 2008.

[13] J. Li and J. Shen, "Existence results for second-order impulsive boundary value problems on time scales," Nonlinear Analysis. Theory, Methods \& Applications A, vol. 70, no. 4, pp. 1648-1655, 2009.

[14] J.-P. Sun, "Twin positive solutions of nonlinear first-order boundary value problems on time scales," Nonlinear Analysis. Theory, Methods \& Applications A, vol. 68, no. 6, pp. 1754-1758, 2008.

[15] R. P. Agarwal, M. Bohner, and D. O'Regan, "Time scale boundary value problems on infinite intervals," Journal of Computational and Applied Mathematics, vol. 141, no. 1-2, pp. 27-34, 2002, Dynamic equations on time scales.

[16] H. Chen, H. Wang, Q. Zhang, and T. Zhou, "Double positive solutions of boundary value problems for $p$-Laplacian impulsive functional dynamic equations on time scales," Computers \& Mathematics with Applications, vol. 53, no. 10, pp. 1473-1480, 2007.

[17] R. Ma and H. Luo, "Existence of solutions for a two-point boundary value problem on time scales," Applied Mathematics and Computation, vol. 150, no. 1, pp. 139-147, 2004.

[18] D. R. Anderson, G. S. Guseinov, and J. Hoffacker, "Higher-order self-adjoint boundary-value problems on time scales," Journal of Computational and Applied Mathematics, vol. 194, no. 2, pp. 309-342, 2006.

[19] Advances in Dynamic Equations on Time Scales, Birkhäuser, Boston, Mass, USA, 2003.

[20] R. P. Agarwal, M. Meehan, and D. O'Regan, Fixed Point Theory and Applications, vol. 141, Cambridge University Press, Cambridge, UK, 2001.

[21] D. J. Guo and V. Lakshmikantham, Nonlinear Problems in Abstract Cones, vol. 5, Academic Press, San Diego, Calif, USA, 1988. 


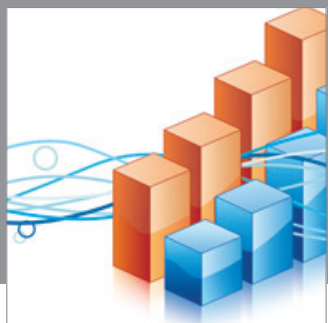

Advances in

Operations Research

mansans

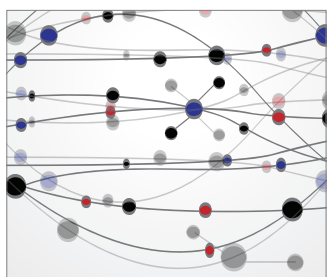

The Scientific World Journal
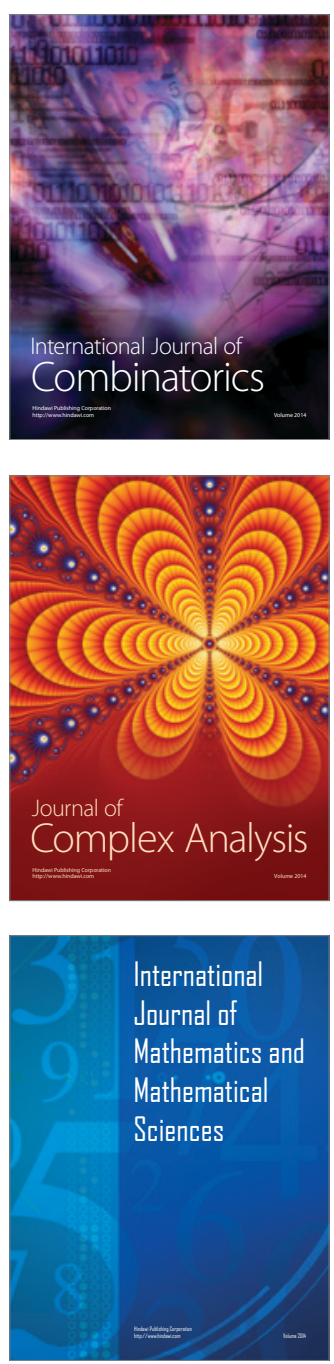
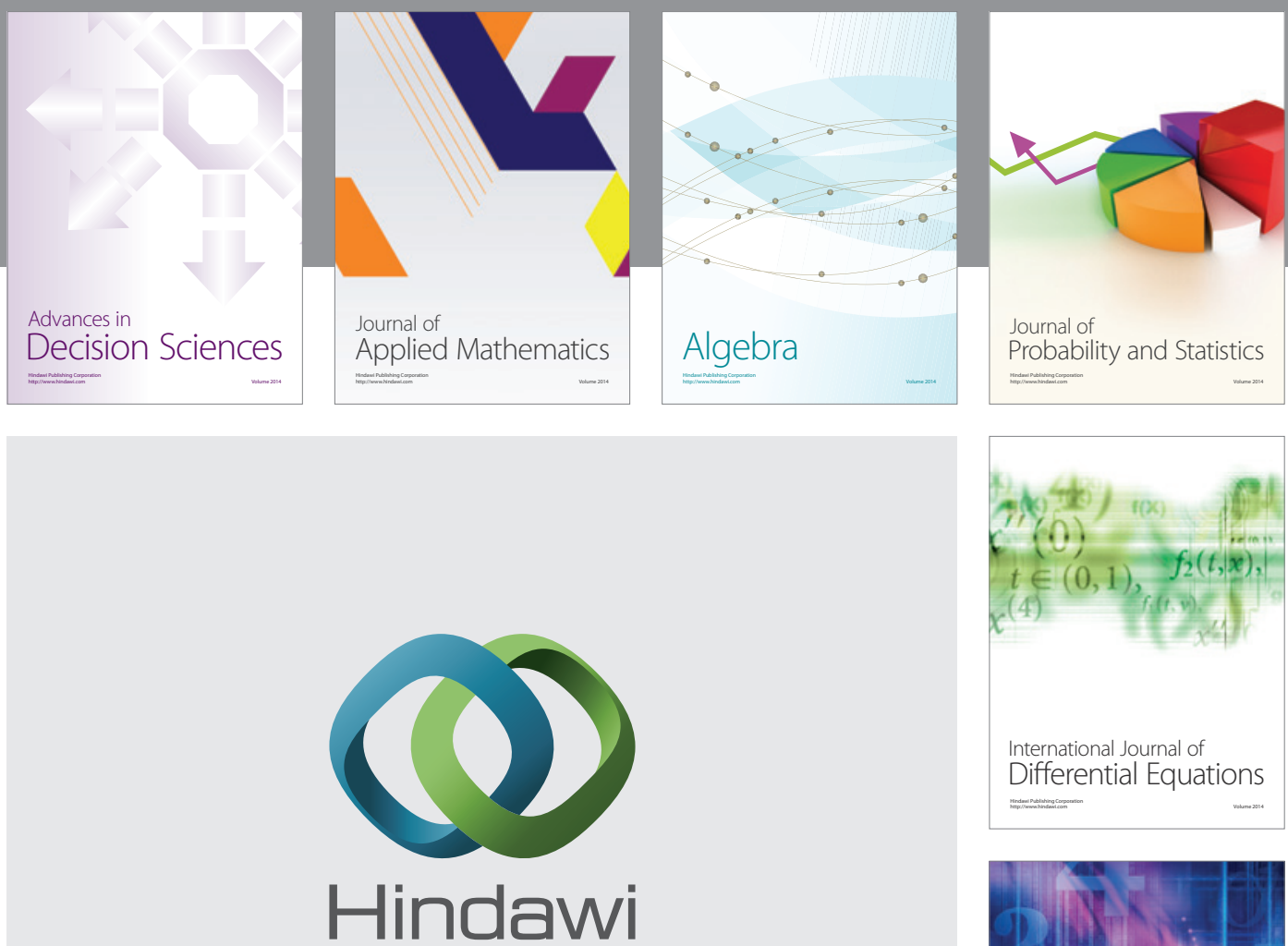

Submit your manuscripts at http://www.hindawi.com
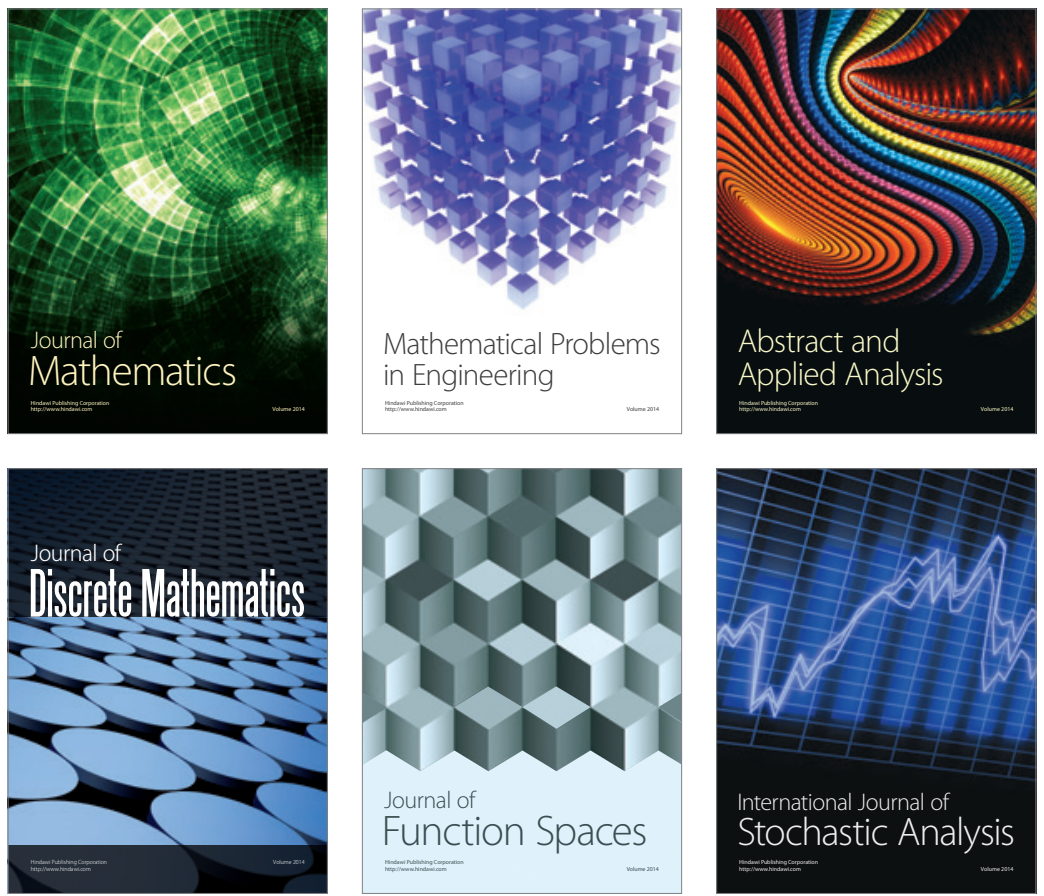

Journal of

Function Spaces

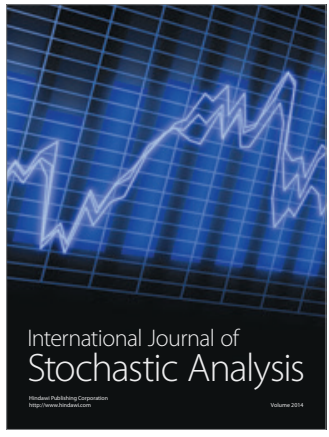

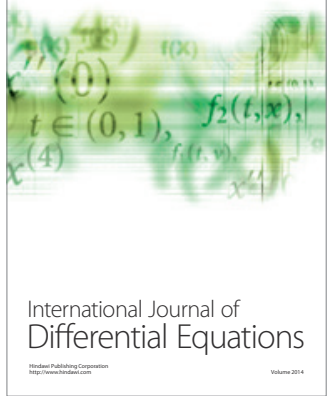
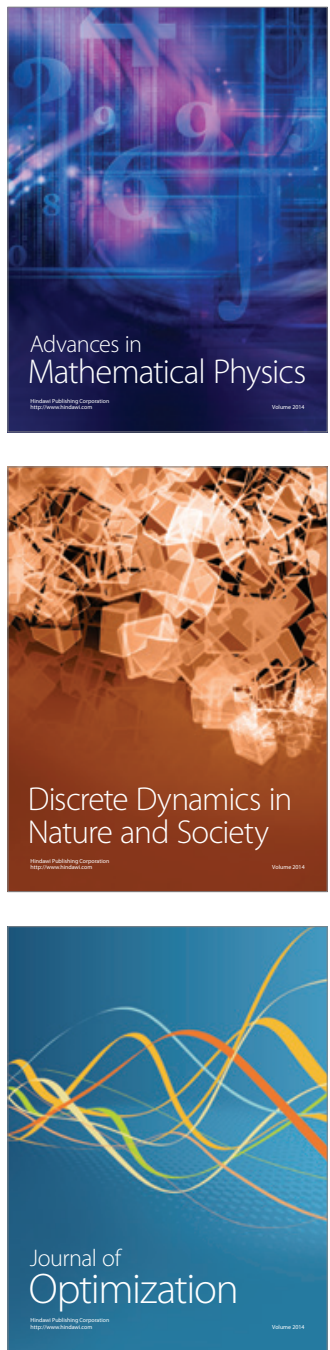